\title{
Preparation and Radical Polymerization of $p$-Vinylphenoxyl Polytetrahydrofuran Macromer
}

\author{
Ryuzo Asami, Mikio TAKaKI, Keizo KYUDA, \\ and Eiji ASAKURA \\ Department of Synthetic Chemistry, Nagoya Institute of Technology, \\ Gokiso-cho, Showa-ku, Nagoya 466, Japan
}

(Received August 31, 1982)

\begin{abstract}
In order to obtain a polytetrahydrofuran macromer, living polytetrahydrofuran was coupled with sodium $p$-vinylphenoxide prepared by the reaction of $p$-hydroxystyrene with sodium tert-butoxide. The resulting $p$-vinylphenoxyl polytetrahydrofuran macromer had one $p$ vinylphenoxyl group per molecule and a high monodispersity $\left(M_{w} / M_{n}=1.1\right)$. The macromer was polymerized by $\alpha, \alpha^{\prime}$-azobisisobutyronitrile in benzene at $60^{\circ} \mathrm{C}$. The maximum degree of polymerization of the poly-macromer was 20 under the polymerization conditions employed. With higher initiator concentration, the conversion increased and the degree of polymerization of the polymacromer became lower. The pure poly-macromer was obtained from a mixture of polymerized and unpolymerized macromers by selective solvent extraction with isopropyl alcohol.
\end{abstract}

KEY WORDS Living Polytetrahydrofuran / Sodium $p$-Vinylphenoxide /

Coupling Reaction / p-Vinylphenoxyl Polytetrahydrofuran / Macromer /

Radical Polymerization /

The synthesis and application of macromers have increasingly taken on interest and the production of a variety of macromers has been reported during the last seven years.

In regard to the synthesis of polytetrahydrofuran (PTHF) macromers, three papers have been published to date. We have already reported the synthesis and radical polymerization of $p$-vinylbenzyloxyl PTHF macromer ${ }^{1}$ obtained by the coupling reaction of living PTHF with sodium $p$ vinylbenzyloxide. Sierra-Vargas et al. synthesized a PTHF macromer by the cationic polymerization of THF using methacryloyl hexafluoroantimonate as an unsaturated initiator ${ }^{2}$ and more recently prepared a PTHF macromer by the reaction of living PTHF with potassium $p$-isopropenylbenzyloxide. ${ }^{3}$

In this study, another method was attempted in preparing a similar type of PTHF macromer and involved the coupling reaction of living PTHF with sodium $p$-vinylphenoxide (VPO-Na) as a nucleophilic deactivator. It was found that VPO-Na could be prepared more easily than sodium $p$-vinylbenzyloxide because of the high acidity of a phenolic proton. The reaction of living PTHF with
VPO-Na was successful in preparing the macromer to a high degree of purity. The PTHF macromer obtained was polymerized by a radical initiator in benzene.

\section{EXPERIMENTAL}

\section{Materials}

A solution of sodium tert-butoxide $(t-\mathrm{BuONa})$ was obtained by the reaction of tert-butyl alcohol with sodium metal in THF. $p$-Hydroxystyrene ( $p$-HS) was prepared by the base-catalyzed hydrolysis of $p$-acetoxystyrene (Maruzen Oil Co.) and metalated with $t$-BuONa in THF to give sodium $p$-vinylphenoxide (VPO-Na). The synthesis of pure triethyloxonium tetrafluoroborate $\left(\mathrm{Et}_{3} \mathrm{OBF}_{4}\right)$ and the preparation of its solutions were performed in the vacuum system described previously. ${ }^{4} p-(2-\mathrm{Hy}-$ droxyethoxy)styrene regarded as a model compound of $p$-vinylphenoxyl PTHF (VPO-PTHF) macromer was synthesized according to the method reported previously. ${ }^{5}$ THF was purified by an available method for the preparation of the living polymer. 


\section{R. AsAmi et al.}

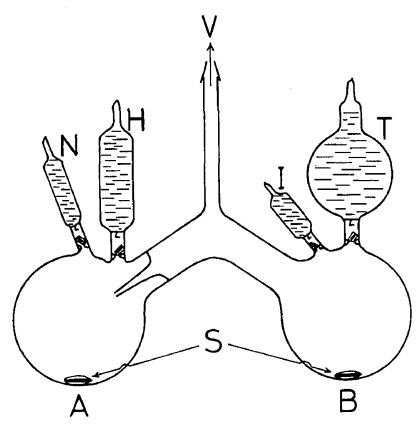

Figure 1. Apparatus for the preparation of VPOPTHF. Symbols are shown in the text.

\section{Coupling Reaction}

The coupling reaction was carried out using the apparatus shown in Figure 1. The apparatus was evacuated on a high vacuum line, sealed off from the line, and placed in an ice bath. The $\mathrm{Et}_{3} \mathrm{OBF}_{4}$ solution $\left(0.13\right.$ or $0.135 \mathrm{~mol}^{-1}$ in 1,2-dichloroethane) in ampule $I$ was then transferred to reactor $B$ and the THF in ampule $T$ was introduced into reactor B so as to start the cationic polymerization of THF. During the polymerization of THF, the $p$-HS solution in ampule $\mathrm{H}$ and the $t-\mathrm{BuONa}$ solution in ampule $\mathrm{N}$ were introduced into reactor A. At the termination of an appropriate period of polymerization, the living PTHF solution in reactor B was added dropwise to the VPO-Na solution with vigorous stirring by stirrer $\mathrm{S}$ to obtain a highly monodisperse macromer. The reaction mixture was pured into a large amount of a methanolwater $(3: 1, \mathrm{v} / \mathrm{v})$ mixture at $0^{\circ} \mathrm{C}$. The precipitated polymer was filtered, purified by reprecipitation from the THF-methanol-water $(3: 1, \mathrm{v} / \mathrm{v})$ system, and then dried in vacuo.

\section{Characterization}

The content of the $p$-vinylphenoxyl group in PTHF macromer was spectroscopically determined using the molar extinction coefficient of $p$-(2hydroxyethoxy)styrene $\left(\varepsilon_{\max }=2.23 \times 10^{4} \quad 1 \mathrm{~mol}^{-1}\right.$ $\mathrm{cm}^{-1}$ at $\lambda_{\max }=262 \mathrm{~nm}$ in THF), since the UV absorption of PTHF at $262 \mathrm{~nm}$ was negligibly small in comparison with that of the $p$-vinylphenoxyl group. The conversion of VPO-PTHF in the radical polymerization was evaluated from the GPC curve with an RI detector or from the decrease in UV absorption at $262 \mathrm{~nm}$.

The intrinsic viscosity of the macromers was measured in toluene at $30.0^{\circ} \mathrm{C}$ using Ubbelohde type viscometer. The number-average molecular weights of the macromers were determined with a Hitachi Perkin-Elmer 115 vapor pressure osmometer using benzene as the solvent at $41^{\circ} \mathrm{C}$. The GPC was recorded on a Toyo Soda high-speed liquid chromatograph Model HLC 802UR (detectors: UV and RI) connected to two GMH columns or $\mathrm{G} 2000 \mathrm{H}$ and $\mathrm{G} 3000 \mathrm{H}$ columns $(61 \mathrm{~cm} \times 2$, Toyo Soda). The former columns were used for the polymerized macromer and the latter for the macromer. THF was used as the eluent and the flow rate was $1 \mathrm{ml} \mathrm{min}^{-1}$. The molecular weight distributions $\left(M_{w} / M_{n}\right)$ of the polymers were calculated from their GPC traces using a calibration curve of standard PTHF samples.

\section{RESULTS AND DISCUSSION}

\section{Preparation of VPO-PTHF}

The coupling reaction of VPO-Na with living PTHF was carried out under reaction conditions chosen so as to obtain a high degree of coupling and a highly monodisperse macromer. The mole ratios of $\left[\mathrm{Et}_{3} \mathrm{OBF}_{4}\right]$ to $[t-\mathrm{BuONa}]$ and of $[t-\mathrm{BuONa}]$ to $[p-$ HS] were chosen to be less than unity, or otherwise the resulting VPO-PTHF macromer would have been contaminated by PTHF having no $p$ vinylphenoxyl groups at the chain ends. In order to prepare a highly monodisperse PTHF macromer, the living polymerization of THF was terminated with VPO-Na before conversion in the bulk polymerization of THF increased to more than about $7 \mathrm{wt} \%$. It has already been suggested that the chain transfer of the active ends of the living PTHF to ether groups in PTHF is not negligible at a THF conversion as low as $8 \mathrm{wt} \%{ }^{4,6-8}$

The detailed reaction conditions and the results of the coupling reaction are summarized in Table I. The VPO-PTHF macromers thus obtained were characrerized as shown in Table II. The value of $\mathrm{II} / \mathrm{O}^{+}$in Table I represents the mole ratio of oxonium ions of living PTHF to those of $\mathrm{Et}_{3} \mathrm{OBF}_{4}$ since the living PTHF is coupled with VPO-Na almost quantitatively as will be demonstrated below. Thus, the $\mathrm{II} / \mathrm{O}^{+}$ratio corresponds to the initiator efficiency, and the relatively low value in Exptl no. M05-1 might result from a shorter polymerization time. These results are consistent with the fact that the concentration of propagating spe- 
Table I. Coupling reactions of living PTHF with VPO-Na under various conditions

\begin{tabular}{|c|c|c|c|c|c|c|c|c|c|}
\hline \multirow{3}{*}{ Exptl no. } & \multicolumn{4}{|c|}{ Living $\mathrm{PTHF}^{\mathrm{a}}$} & \multicolumn{2}{|c|}{ VPO-Na } & \multirow{3}{*}{$\begin{array}{c}\text { Coupling } \\
\text { reaction }^{\mathrm{d}} \\
\mathrm{ONa} / \mathrm{O}^{+}\end{array}$} & \multirow{2}{*}{\multicolumn{2}{|c|}{$\begin{array}{c}\text { Yield } \\
\text { of } \\
\text { polymer }\end{array}$}} \\
\hline & \multirow{2}{*}{$\frac{\mathrm{THF}}{\mathrm{cm}^{3}}$} & \multirow{2}{*}{$\frac{\mathrm{O}^{+\mathrm{b}}}{\mathrm{mmol}}$} & \multirow{2}{*}{$\frac{\text { Time }}{\min }$} & \multirow{2}{*}{$\mathrm{II}^{\prime c} / \mathrm{O}^{+}$} & \multirow{2}{*}{$\frac{p \text {-HS }}{\mathrm{mmol}}$} & \multirow{2}{*}{$\frac{t-\mathrm{BuONa}}{\mathrm{mmol}}$} & & & \\
\hline & & & & & & & & $\mathrm{g}$ & $\%$ e \\
\hline M05-1 & 65.9 & 0.690 & 30 & 0.61 & 1.03 & 0.896 & 1.29 & 1.92 & 3.12 \\
\hline M10-1 & 68.3 & 0.326 & 60 & 0.76 & 0.494 & 0.414 & 1.29 & 2.65 & 4.36 \\
\hline-2 & 68.4 & 0.323 & 60 & 0.78 & 0.506 & 0.414 & 1.28 & 2.61 & 4.30 \\
\hline-3 & 68.2 & 0.346 & 60 & 0.83 & 0.512 & 0.432 & 1.25 & 3.02 & 4.99 \\
\hline-4 & 70.8 & 0.479 & 60 & 0.77 & 0.720 & 0.596 & 1.24 & 3.89 & 6.18 \\
\hline-5 & 72.4 & 0.483 & 60 & 0.81 & 0.726 & 0.601 & 1.24 & 3.65 & 5.68 \\
\hline M22-1 & 69.4 & 0.346 & 120 & 0.83 & 0.519 & 0.445 & 1.29 & 6.51 & 10.6 \\
\hline
\end{tabular}

a Polymerization temp, $0^{\circ} \mathrm{C}$.

b Amount of initiator $\left(\mathrm{Et}_{3} \mathrm{OBF}_{4}\right)$.

c $\mathrm{II}^{\prime}$ is the polymer yield in mmol calculated using mol wt (II in Table II).

d Reaction time, $40 \mathrm{~min}$; temp, $0^{\circ} \mathrm{C}$.

e Based on THF fed.

Table II. Characterization of the VPO-PTHF macromer

\begin{tabular}{|c|c|c|c|c|c|c|c|}
\hline \multirow[b]{2}{*}{ Exptl no. ${ }^{a}$} & \multirow{2}{*}{$\frac{[\eta]}{\mathrm{dl} \mathrm{g}^{-1}}$} & \multicolumn{4}{|c|}{ Mol wt $t^{b} \times 10^{-3}$} & \multirow{2}{*}{$\begin{array}{c}\mathrm{C}=\mathrm{C} / \\
\text { molecule }^{\mathrm{c}} \\
(\mathrm{I} / \mathrm{II})\end{array}$} & \multirow[b]{2}{*}{$M_{w} / M_{n}^{\mathrm{d}}$} \\
\hline & & Visc. & GPC & $\begin{array}{l}\text { VPO } \\
\text { (I) }\end{array}$ & $\begin{array}{c}\mathrm{UV}(\mathrm{C}=\mathrm{C}) \\
\text { (II) }\end{array}$ & & \\
\hline M05-1 & 0.171 & 3.4 & 4.1 & 4.5 & 4.6 & 0.98 & $1.0_{5}$ \\
\hline M10-1 & - & - & 11 & 9.8 & 10.9 & 0.90 & $1.1_{2}$ \\
\hline-2 & 0.291 & 8.1 & 9.8 & 9.6 & 10.5 & 0.91 & $1.1_{0}$ \\
\hline-3 & - & - & 9.6 & 9.9 & 10.4 & 0.95 & $1.0_{7}$ \\
\hline-4 & 一 & - & 9.7 & 9.6 & 10.5 & 0.91 & $1.1_{5}$ \\
\hline-5 & - & - & 9.0 & 8.7 & 9.4 & 0.93 & $1.1_{2}$ \\
\hline M22-1 & 0.532 & 22.5 & 24.7 & - & 22 & $1.12^{\mathrm{e}}$ & $1.2_{5}$ \\
\hline
\end{tabular}

a Experimental number is the same as in Table I.

b Molecular weights of VPO-PTHF determined by intrinsic viscosity, GPC, VPO, and UV (end group analysis) methods.

c The number of $p$-vinylphenoxyl groups per VPO-PTHF molecule.

d Calculated from GPC traces using a calibration curve of standard PTHF samples.

e Evaluated by molecular weights based on GPC and UV methods.

cies of PTHF is maximum at a polymerization time of about $1 \mathrm{~h} .{ }^{9}$ The molecular weights of the VPOPTHF macromers were determined by four methods including intrinsic viscosity, vapor pressure osmometry (VPO), GPC, and end-group analysis (UV measurement of $\mathrm{C}=\mathrm{C}-(-\mathrm{O}-$ ). Consistent values were obtained by these four independent methods. In particular, a comparison of the molecular weights by the VPO method with those by end-group analysis supports the extent of coupling of the living PTHF with VPO-Na and also the number of $p$-vinylphenoxyl groups per VPO-PTHF molecule just as expected. Thus, the II/I ratios in Table II suggest that the extent of coupling of the living PTHF is almost $100 \%$, and that a VPOPTHF molecule has a $p$-vinylphenoxyl group in each chain. The molecular weight distributions of the VPO-PTHF macromers obtained were as low as 1.1, as given in Table II.

The UV spectrum of VPO-PTHF, as shown in 
Figure 2, had a peak at $262 \mathrm{~nm}$ and was quite similar to that of $p$-(2-hydroxyethoxy)styrene which may be regarded as a model compound for VPOPTHF. The coincidence of the UV spectra of the macromer with the model compound possibly stems

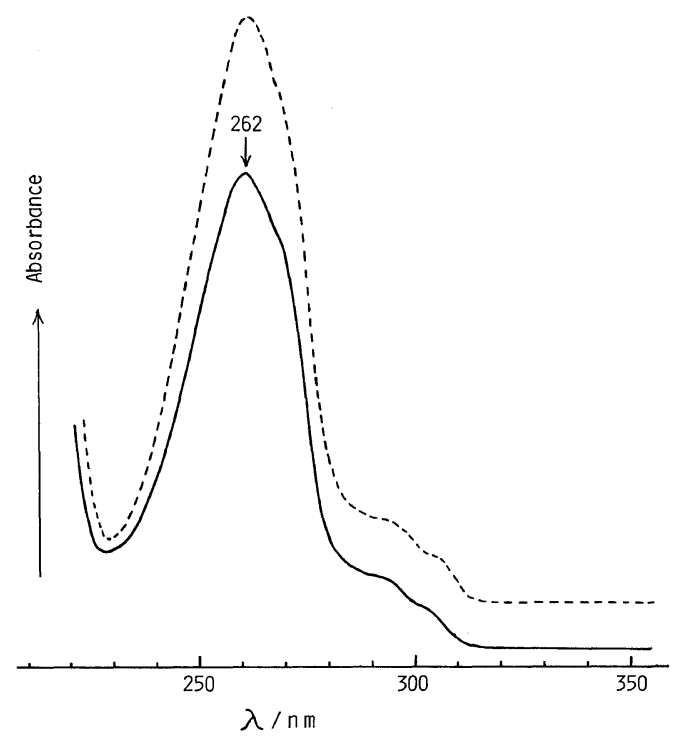

Figure 2. UV spectra of VPO-PTHF (-, $1.83 \times$ $\left.10^{-4} \mathrm{moll}^{-1}\right)$ and its model compound (---, $p$-(2hydroxyethoxy)-styrene, $1.57 \times 10^{-4} \mathrm{moll}^{-1}$ ) in THF.

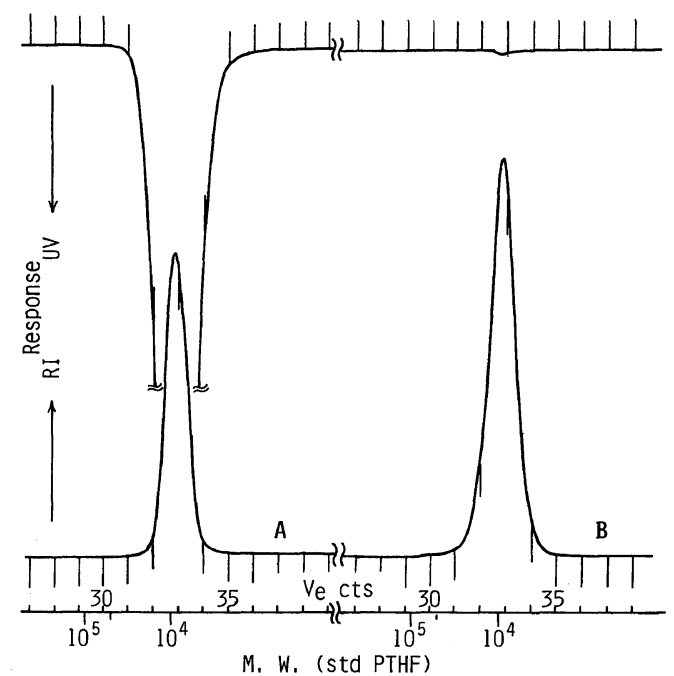

Figure 3. GPC curves (UV and RI detectors) of living PTHF terminated with VPO-Na (A) or alkaline methanol (B). from the fact that the absorption of PTHF over the UV range is negligibly small in comparison with that of a $p$-vinylphenoxyl group. Figure 3 shows the GPC curves of the living PTHFs terminated with VPO-Na and alkaline methanol. It can be seen that the peak of the VPO-PTHF macromer by a UV detector is extremely higher than that of PTHF as expected. Furthermore, both the GPC peaks (by an RI detector) of VPO-PTHF and PTHF are symmetrical, sharp, and similar to each other.

These observations support the conclusion that the reaction of the living PTHF with VPO-Na is an ideal coupling reaction for the preparation of the PTHF macromer as well as the $p$-vinylbenzyloxyl PTHF macromer. ${ }^{1}$

\section{Radical Polymerization of VPO-PTHF}

VPO-PTHF macromers prepared were polymerized using various amounts of AIBN in benzene at $60^{\circ} \mathrm{C}$. The results are listed in Table III. As the amount of added AIBN increased, the conversion of the macromer to poly(VPO-PTHF) increased whereas the degree of polymerization (D.P.) of poly(VPO-PTHF) decreased. This dependence of the conversion and D.P. on the amount of added initiator was more pronounced with a lesser amount of AIBN, as shown in Figure 4. Figure 4 also shows that above $1 \mathrm{wt} \%$ of AIBN, the conversion was 70 to $90 \%$ whereas the D.P. was 5 to 3 and when the

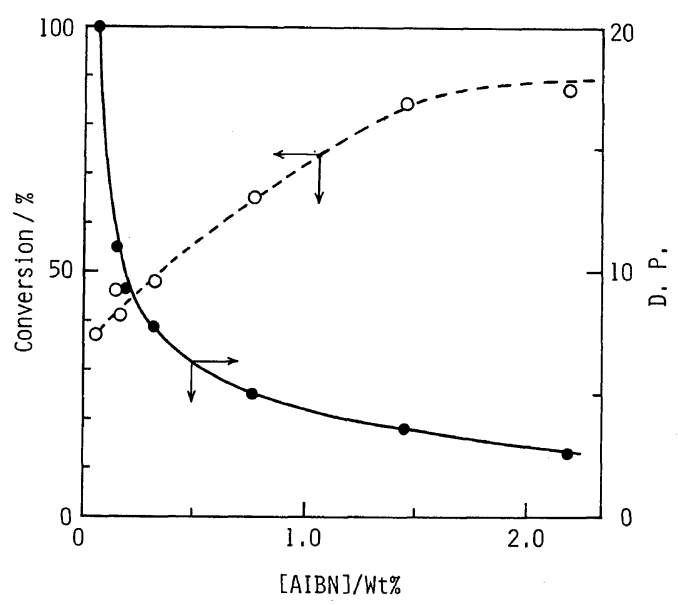

Figure 4. Plots for the various amounts of added AIBN $v s$. conversion of VPO-PTHF $\left(M_{n}=1.0 \times 10^{4}\right)$ to poly(VPO-PTHF) and the degree of polymerization of poly(VPO-PTHF). 
Table III. Radical polymerization of VPO-PTHF ${ }^{\mathrm{a}}$

\begin{tabular}{|c|c|c|c|c|c|c|c|c|c|}
\hline \multirow{3}{*}{ Exptl no. } & \multicolumn{3}{|c|}{ VPO-PTHF } & \multicolumn{2}{|c|}{ AIBN } & \multirow{3}{*}{$\frac{\text { Benzene }}{\mathrm{mg}}$} & \multicolumn{2}{|c|}{ Conversion } & \multirow{3}{*}{ D.P. ${ }^{c}$} \\
\hline & \multirow{2}{*}{$\begin{array}{c}\text { Sample }^{\mathrm{b}} \\
\text { no. }\end{array}$} & \multicolumn{2}{|r|}{$\begin{array}{c}{[\mathrm{C}=\mathrm{C}]} \\
\times 10^{2}\end{array}$} & \multicolumn{2}{|r|}{$\begin{array}{c}{[\mathrm{AIBN}]} \\
\times 10^{2}\end{array}$} & & \multirow{2}{*}{$\frac{\mathrm{UV}}{\%}$} & \multirow{2}{*}{$\frac{\text { GPC }}{\%}$} & \\
\hline & & $\mathrm{mg}$ & $\mathrm{moll}^{-1}$ & $\mathrm{mg}$ & $\mathrm{moll}^{-1}$ & & & & \\
\hline P-1 & M05-1 & 378 & 11 & 3.07 & 2.2 & 382 & 64 & 59 & 11 \\
\hline-2 & M10-3 & 410 & 4.4 & 0.41 & 0.28 & 409 & 37 & 37 & 20 \\
\hline-3 & M10-2 & 492 & 4.3 & 1.50 & 0.83 & 510 & 43 & 46 & 11 \\
\hline-4 & M10-1 & 785 & 4.1 & 2.75 & 0.94 & 843 & 41 & 41 & 9.2 \\
\hline-5 & M10-3 & 775 & 4.3 & 5.19 & 1.8 & 799 & 49 & 48 & 7.7 \\
\hline-6 & M10-2 & 492 & 4.3 & 7.76 & 4.3 & 503 & 68 & 65 & 5.0 \\
\hline-7 & M10-2 & 496 & 4.4 & 14.6 & 8.2 & 493 & 82 & 84 & 3.6 \\
\hline-8 & M10-1 & 499 & 3.9 & 24.0 & 12 & 580 & 85 & 87 & 2.6 \\
\hline-9 & M22-1 & 401 & 2.0 & 3.26 & 2.2 & 405 & 54 & 66 & 4.6 \\
\hline
\end{tabular}

a Temp, $60^{\circ} \mathrm{C}$; time, $22 \mathrm{~h}$.

b For example, VPO-PTHF of M10-2 is VPO-PTHF obtained in experimental number M10-2 in Table I.

c Degree of polymerization estimated from GPC using a calibration curve of standard PTHF.

amount of AIBN was least $(0.05 \mathrm{wt} \%)$, the D.P. was 20 under the polymerization conditions used. A similar relationship between the amount of initiator and conversion of the macromer or the D.P. of poly-macromers has also been found in the case of the radical polymerization of a $p$-vinylbenzyloxyl PTHF macromer. ${ }^{1}$

Table III shows that the conversion of the macromer and the D.P. of poly(VPO-PTHF) are not affected by the molecular weight of VPOPTHF. The relatively high D.P. in Exptl no. P-1 may not be due to the low molecular weight of the macromer (M05-1) but to the high concentration of the macromer, as will be described below.

In the previous paper, ${ }^{1}$ we discussed the low D.P. of poly ( $p$-vinylbenzyloxyl PTHF) on the basis of the following considerations. In the radical polymerization of macromers, the rate of propagation and termination would be considerably suppressed by steric hindrance and the decrease in the mobility of macromers but not the diffusion controlled rates of chain transfer to the solvents and to the ether groups in the PTHF chain. However, in a more recent kinetic study ${ }^{10}$ on the radical polymerization of the PTHF macromer in dilute solutions, we found that both the polymerization rate and the D.P. of the poly-macromer are independent of the molecular weight of the macromer. Moreover, the D.P. of the poly-macromer can be estimated from the following well-known equation ${ }^{11}$ derived kinetically,

$$
\begin{aligned}
& \frac{1}{P_{\mathrm{n}}}=A \frac{R_{\mathrm{p}}}{[\mathrm{M}]^{2}}+C_{\mathrm{M}}+C_{\mathrm{I}} \frac{[\mathrm{I}]}{[\mathrm{M}]}+C_{\mathrm{S}} \frac{[\mathrm{S}]}{[\mathrm{M}]}+C_{\mathrm{P}} \frac{[\mathrm{P}]}{[\mathrm{M}]} \\
& A=\frac{k_{\mathrm{tc}}+2 k_{\mathrm{td}}}{2 k_{\mathrm{p}}{ }^{2}} \quad R_{\mathrm{p}}=k_{\mathrm{p}}\left(\frac{2 f k_{\mathrm{d}}}{k_{\mathrm{tc}}+k_{\mathrm{td}}}\right)^{1 / 2}[\mathrm{I}]^{1 / 2}[\mathrm{M}],
\end{aligned}
$$

and the (D.P.)s in Expt1 no. P-1 and P-5 were calculated to be 13 and 3 , respectively, using the following constants ${ }^{11}$ in the polymerization of styrene by AIBN at $60^{\circ} \mathrm{C}: k_{\mathrm{tc}}=3.6 \times 10^{7} 1 \mathrm{~mol}^{-1} \mathrm{~s}^{-1}$; $k_{\mathrm{td}}=0 ; k_{\mathrm{p}}=1761 \mathrm{~mol}^{-1} \mathrm{~s}^{-1} ; f=0.8 ; k_{\mathrm{d}}=8.5 \times 10^{-6}$ $\mathrm{s}^{-1} ; C_{\mathrm{M}}=6 \times 10^{-5} ; C_{\mathrm{I}}=0 ; C_{\mathrm{S}}=1.8 \times 10^{-6} ; C_{\mathrm{P}}=$ $2.0 \times 10^{-5}$ (constant for polyoxyethylene). The calculated (D.P.)s of 13 and 3 approximately agree with those observed, 11 and 7.7, respectively, although the calculation was performed using the rate constant of styrene and the initial concentration of the macromer as $[\mathrm{M}]$. Therefore, the low D.P. of poly(VPO-PTHF) is not ascribed to the high molecular weight of the macromer but is primarily the result of the low concentration of vinyl groups in the macromer.

As shown in Table III, the polymer recovered after the polymerization of VPO-PTHF is usually a mixture of poly(VPO-PTHF) as the main constituent and several percent of the unpolymerized macromer. However, the poly(VPO-PTHF) could 


\section{R. Asami et al.}

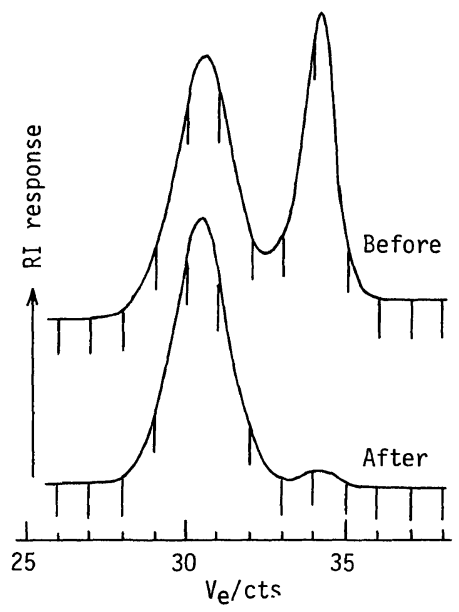

Figure 5. Isolation of poly(VPO-PTHF) from the recovered polymer in experimental number P-5 by extraction with isopropyl alcohol.

easily be isolated from this mixture by fractionation with isopropyl alcohol. That is, the recovered polymer was dissolved in hot $\left(80^{\circ} \mathrm{C}\right)$ isopropyl alcohol and then the solution was slowly cooled to room temperature with stirring. The poly(VPO-PTHF) precipitated out gradually and filtered, washed with the alcohol, and then dried in vacuo. The poly(VPOPTHF) thus obtained scarcely contained any unpolymerized macromer as shown in Figure 5. Thus, by this method, pure multibranched polymers can be obtained even at the low macromer conversion.

Acknowledgment. We express our appreciation for the generous gift of acetoxystyrene from Maruzen Oil Company.

\section{REFERENCES}

1. R. Asami, M. Takaki, K. Kita, and E. Asakura, Polym. Bull., 2, 713 (1980).

2. J. Sierra-Vargas, J. G. Zilliox, P. Rempp, and E. Franta, Polym. Bull., 3, 83 (1980).

3. J. Sierra-Vargas, P. Masson, G. Beinert, P. Rempp, and E. Franta, Polym. Bull., 7, 277 (1982).

4. R. Asami, M. Takaki, Y. Harima, and S. Tsuzuki, Polym. J., 13, 141 (1981).

5. R. Asami and E. Ando, Polym. Prepr. Jpn., 28, 5 (1979).

6. E. Franta, F. Afshar-Taromi, and P. Rempp, Makromol. Chem., 177, 2191 (1976).

7. S. Penczek, P. Kubisa, and K. Matyjaszewski, $A d v$. Polym. Sci., 37 (1980).

8. T. Fujimoto, M. Kawahashi, M. Nagasawa, and A. Takahashi, Polym. J., 11, 193 (1979).

9. T. Saegusa and S. Matsumoto, J. Macromol. Sci., A4, 873 (1970).

10. R. Asami, M. Takaki, K. Kyuda, and N. Sukenaga, Polym. Prepr. Jpn., 30, 856 (1981); to be published.

11. J. C. Masson, R. Korus, K. F. O'Driscoll, and Lewis J. Young, "Polymer Handbook," 2nd ed, II-1-52, 57-104, J. Brandrup and E. H. Immergut Ed., John Wiley, New York, N. Y., 1975. 